\title{
Coronary Atherosclerosis and Malignancies in Diabetic Patients
}

\author{
Joseph C. Lee ${ }^{a, b}$ David G. Lyallc Yong S. Wee ${ }^{b, d}$ \\ aDepartment of Medical Imaging, The Prince Charles Hospital, Brisbane, QLD, Australia; ${ }^{b}$ Faculty of Medicine, \\ University of Queensland, Brisbane, QLD, Australia; 'School of Health Sciences, University of Newcastle, Newcastle, \\ NSW, Australia; ${ }^{d}$ Department of Cardiology, The Prince Charles Hospital, Brisbane, QLD, Australia
}

\section{Dear Editor,}

We concur with the conclusions of Azencot et al. [1] that it is very hard to draw a genuine link between coronary artery atherosclerosis (and its extent as well as characteristics) and malignancies in diabetic patients. On one hand, it is surprising given the link between diabetes and malignancies of various types. Such examples are covered in their article, and there has also been no shortage of even more recent literature in this domain [2-4]. There has also been suggestion of common molecular signalling pathways for diabetes and lung cancer with potential implications on therapeutic approaches [5].

However, it is somewhat unsurprising given that diabetes and the concurrence of haemodynamically significant coronary atherosclerosis were not associated with an increased incidence of malignancies (as shown on the attenuation correction computed tomography images) not known previously in a series of patients assessed with myocardial perfusion scintigraphy [6]. In that series, there were 3,758 patients assessed with myocardial perfusion scintigraphy. Of those, $436(11.6 \%)$ were found to have ischaemia. Amongst these, 25 had incidental findings ( $p=0.42)$. In the series, $201(5.3 \%)$ had an infarct and
$100(2.7 \%)$ had infarction with peri-infarctional ischaemia. Of these, 18 and 5 patients had incidental findings ( $p=0.39$ and $p=0.38$, respectively). Similarly, 34 patients were found to have infarction with ischaemia at another site altogether, and of these, only one was found to have a significant incidental finding $(p=0.38)$.

Regardless of coronary atherosclerosis status, findings suggestive of significant pathology - especially malignancy - warrant further evaluation. The importance and potential clinical value of any modality of coronary disease imaging (anatomical or functional) and further investigation of suspicious incidental findings cannot be underestimated. It is imperative to ensure suspicious incidental findings are given sufficient credence and thoroughly investigated promptly to avoid adverse clinical outcomes [7].

\section{Conflict of Interest Statement}

Dr. Lee has received travel expenses for attending meetings of his specialty College's Continuing Professional Development Committee, of which he is a member. He has received no other financial or nonfinancial benefit from this body. Mr. Lyall has nothing to disclose. Dr. Wee has nothing to disclose.
Correspondence to:

Joseph C. Lee, joseph.lee@ health.qld.gov.au 


\section{Funding Sources}

There were no funding sources.

\section{References}

1 Azencot M, Lewis BS, Halon DA. Relation between baseline coronary atherosclerotic status, cardiovascular events, and malignancies in type 2 diabetics: a long-term prospective cohort study. Cardiology. 2021;146(4):41925.

2 Wu BU. Diabetes and pancreatic cancer: recent insights with implications for early diagnosis, treatment and prevention. Curr Opin Gastroenterol. 2021;37(5):539-43.

3 Tseng $\mathrm{CH}$. The relationship between diabetes mellitus and gastric cancer and the potential benefits of metformin: an extensive review of the literature. Biomolecules. 2021;11(7):1022.

\section{Author Contributions}

J.C.L. (60\%), D.G.L. (25\%), and Y.S.W. (15\%) contributed to the conception or design of the work; and the acquisition, analysis, or interpretation of data for the work.
4 Shi N, Shi Y, Xu J, Si Y, Yang T, Zhang M, et al. SGLT-2i and risk of malignancy in type 2 diabetes: a meta-analysis of randomized controlled trials. Front Public Health. 2021;9: 668368.

5 Raguraman R, Srivastava A, Munshi A, Ramesh R. Therapeutic approaches targeting molecular signaling pathways common to diabetes and lung diseases and cancer. Adv Drug Deliv Rev. 2021 Aug 8. Epub ahead of print.
6 Lee JC, Delaney FT. Prevalence and clinical significance of incidental findings on CT attenuation correction for myocardial perfusion imaging. J Nucl Cardiol. 2021 Mar 22. Epub ahead of print.

7 Delaney FT, Gray EL, Lee JC. The importance of appropriate reporting and investigation of incidental findings on computed tomography attenuation correction images during myocardial perfusion scintigraphy. World J Nucl Med. 2019 Jan-Mar;18(1):74-6. 